\title{
MISTURA DE TRÊS ESPÉCIES DE REFLORESTAMENTO NA PRODUÇÃO DE PAINÉIS CIMENTO-MADEIRA ${ }^{1}$
}

\author{
Vânia Aparecida de Sá ${ }^{2}$, Lina Bufalino², Vanessa C. S. Albino ${ }^{3}$, Andréa A. Corrêa ${ }^{4}$, Lourival M. Mendes ${ }^{5}$ e \\ Natália A. Almeida ${ }^{4}$
}

\begin{abstract}
RESUMO - Este trabalho teve por objetivo avaliar a viabilidade técnica de produção de painéis cimentomadeira compostos por diferentes proporções de resíduos das madeiras de cedro-australiano (Toona ciliata M. Roem.), Pinus taeda e clone de híbrido de Eucalyptus grandis x Eucalyptus urophylla, utilizando-se dois processos distintos de cura: vaporização e climatização. Foi utilizado o cimento Portland CP V - ARI/Plus como aglomerante e o cloreto de cálcio como aditivo químico. As partículas de madeira foram previamente tratadas em água fria durante $24 \mathrm{~h}$. Foram considerados os seguintes parâmetros: relação madeira/cimento - 1/2,75; relação água/cimento - 1/2,5 (base peso do cimento); taxa de água de hidratação/cimento - $25 \%$ (base peso do cimento); aditivo químico - 4\% (base peso do cimento); densidade nominal $-1,10 \mathrm{~g} / \mathrm{cm}^{3}$; e dimensões dos painéis -480 × $480 \times 150 \mathrm{~mm}$. Os parâmetros de prensagem foram: temperatura ambiente, pressão específica de $40 \mathrm{kgf} / \mathrm{cm}^{2}$ e tempo de $10 \mathrm{~min}$. O tempo de grampeamento foi de $24 \mathrm{~h}$. As propriedades avaliadas foram absorção de água e inchamento em espessura após 24 h, flexão estática, compressão e ligação interna. O experimento foi instalado segundo um delineamento inteiramente casualizado com três repetições e seis tratamentos, arranjados num esquema fatorial de três composições x dois processos de cura. Os painéis compostos pela mistura de T. ciliata com o clone de Eucalyptus apresentaram valores médios superiores para inchamento em espessura e resultados inferiores para a maioria das propriedades mecânicas. A cura a vapor melhorou as propriedades físicas, mas afetou negativamente a maioria das propriedades mecânicas.
\end{abstract}

Palavras-chave: Cedro-australiano, Cura e Resíduos.

\section{MIXTURE OF THREE REFORESTATION SPECIES ON THE CEMENT-WOOD PANELS PRODUCTION}

\begin{abstract}
The objective of this work was to evaluate the technical feasibility of producing cement-wood panels made up from different proportions of Australian cedar (Toona ciliata M. Roem.), Eucalyptus grandis $x$ Eucalyptus urophylla and Pinus taeda wood wastes using two different curing procedures: acclimation and vaporization. Portland CP V-ARI/Plus cement was used as binder and calcium chloride as additive. The wood particles were previously treated in water for 24 hours. The following parameters were considered for the estimation: wood/cement ratio-1/2.75; water/cement ratio-1/2.5 (based upon cement weight); hydration water/cement ratio-25\% (based upon cement weight); chemical additive - 4\% (based upon cement weight); target density $-1.10 \mathrm{~g} / \mathrm{cm}^{3}$; panel dimensions - $480 \times 480 \times 150 \mathrm{~mm}$. The pressing parameters were the following: room temperature, specific pressure of $40 \mathrm{kgf/ \textrm {cm } ^ { 2 }}$ and a time period of 10 minutes. Clasping time
\end{abstract}

\footnotetext{
${ }^{1}$ Recebido em 04.09.2009 e aceito para publicação em 19.04.2012.

${ }^{2}$ Professora Temporária do Departamento de Agricultura e Ambiente do Instituto Federal do Sudeste de Minas Gerais, Rio Pomba, Brasil. E-mail: <vania_desa@yahoo.com.br>

${ }^{3}$ Pós Graduação em andamento em Ciência e Tecnologia da Madeira pela Universidade Federal de Lavras, UFLA, Brasil. E-mail: <linabufalino@yahoo.com.br>e < deiacor@uaigiga.com.br>.

${ }^{4}$ Mestres em Ciência e Tecnologia da Madeira pela Universidade Federal de Lavras, UFLA, Brasil.E-mail:<vanessa.floresta@ hotmail.com> e $<$ natamarant@gmail.com>.

${ }^{5}$ Professor Adjunto do Departamento de Ciências Florestais da Universidade Federal de Lavras, UFLA, Brasil. E-mail: $<$ lourival@dcf.ufla.br>.
} 
was 24 hours. The properties evaluated were water absorption and thickness swelling after 24 hours, static bending, compression and internal bonding. Experimental statistical design was entirely randomized with three replications and six treatments, arranged in a factorial design of 3 compositions and 2 curing procedures. Panels made from T. ciliata and Eucalyptus grandis $x$ Eucalyptus urophylla presented higher average values for thickness swelling and inferior values for most mechanical properties. Vaporization cure improved physical properties, but negatively influenced most of mechanical properties.

Keywords: Australian cedar, Cure and Residues.

\section{INTRODUÇÃO}

Os painéis cimento-madeira são constituídos por partículas ou fibras de biomassa vegetal, aglomerante do tipo mineral, água e aditivos químicos, sendo estes consolidados sob pressão na temperatura ambiente. São produtos de uso estrutural que apresentam excelente isolamento térmico e acústico, possuem alta resistência ao fogo, aos agentes biodegradadores e à variação de umidade (HERRERA; CLOUTIER, 2008; PAPADOULOS, 2006; LATORRACA; IWAKIRI, 2001).

Alguns fatores limitantes na produção desses painéis são o elevado peso, a lenta velocidade de cura e a incompatibilidade química entre algumas essências florestais e o aglomerante mineral, devido à presença de substâncias químicas (compostos fenólicos, ácidos e açúcares) que podem retardar ou impedir a cura do cimento (SAVASTANO JÚNIOR et al., 2000; SANTOS et al., 2008).

Para viabilizar o uso de espécies com altos teores de extrativos, normalmente se têm extraído as substâncias químicas inibidoras, como os extrativos solúveis em água ou álcali, carboidratos livres etc., por imersão das partículas em água fria por 24 h, imersão em água quente por 6 h imersão em solução de hidróxido de sódio $(\mathrm{NaOH})$ a $1 \%$ (MOSLEMI etal., 1983; SAVASTANO JÚNIOR et al., 2000). Segundo Beraldo et al. (2002), o problema relacionado ao tempo de pega do cimento pode ser minimizado com a aplicação de aditivos químicos e minerais aceleradores da cura.

Okino et al. (2004) avaliaram os efeitos de inibição da cura sobre as propriedades dos painéis cimentomadeira confeccionados a partir da madeira de Hevea brasiliensis, por teste de hidratação das partículas, com e sem fervura, em água quente e com ou sem adição de $\mathrm{CaCl}_{2}$. Os resultados indicaram que a madeira de seringueira in natura foi classificada como de "inibição extrema"; com a adição de $\mathrm{CaCl}_{2}$, no entanto foi classificada como de "baixa inibição". Iwakiri e Prata
(2008), avaliando o efeito do tratamento das partículas de madeira de Eucalyptus em água fria e em água quente sobre as propriedades dos painéis cimento-madeira, concluíram que não há necessidade de tratamento das espécies de E. grandis e E. dunnii.

A cura a vapor, sob pressão atmosférica (temperatura de vapor abaixo de $100^{\circ} \mathrm{C}$ ), é bastante empregada em indústrias de artefatos de concretos pré-fabricados por acelerar o processo de maturação e fornecer alta resistência inicial aos painéis, implicando vantagem econômica, já que possibilita o manuseio dos produtos pouco tempo após a moldagem. Paula et al. (2009) desenvolveram experimentalmente uma câmara a vapor e estudaram os efeitos do tempo ( 3 e 10 dias) e da temperatura $\left(50,60\right.$ e $\left.70{ }^{\circ} \mathrm{C}\right)$ na cura dos painéis. Encontraram efeito significativo da temperatura nas propriedades fisicomecânicas estudadas, sendo $60^{\circ} \mathrm{C}$ a de melhor desempenho.

Em teoria, todo e qualquer material lignocelulósico pode compor os painéis cimento-madeira, entretanto as espécies de coníferas são as madeiras mais empregadas, por apresentarem propriedades, principalmente químicas, compatíveis, que podem ser combinadas com o cimento sem afetar a cura e o seu endurecimento (LATORRACA; IWAKIRI, 2001).

No Brasil, as indústrias de painéis utilizam quantidades significativas de madeiras provenientes de cultivos florestais de pinus e algumas espécies de eucalipto. Para atender ao crescente aumento da demanda por produtos de madeira, é necessário o estudo de espécies alternativas de rápido crescimento que possam diversificar a oferta de matéria-prima e suprir a possível carência das indústrias de base florestal. Nesse contexto, várias espécies florestais têm sido alvo de pesquisas, e algumas têm sido consideradas promissoras para a confecção de painéis cimento-madeira, podendo-se citar: Eremanthus erytropappus - candeia (SANTOS et al., 2008); Hevea brasiliensis - seringueira (OKINO

Revista Árvore, Viçosa-MG, v.36, n.3, p.549-557, 2012 
et al., 2004); Eucalyptus spp. (LATORRACA, 2000; IWAKIRI; PRATA, 2008) - eucalipto; e Parkia gigantocarpa-faveira (LIMA et al., 2010), entre outras.

O cedro-australiano (Toona ciliata M. Roem.), pertencente à importante família Meliaceae, é uma espécie originária das regiões que se estendem desde a Índia até a Austrália. Introduzida no Brasil, principalmente nas regiões Sudeste e Sul da Bahia, apresenta excelentes taxas de crescimento e resistência ao ataque da mariposa de ponta (Hypsipyla grandella), importante praga que afeta os cedros nativos (Cedrela odorata e Cedrela fissilis) e o mogno-brasileiro (Swietenia macrophylla) (BYGRAVE; BYGRAVE, 2005; PINHEIRO et al., 2006).

A madeira de cedro-australiano apresenta diversas possibilidades de uso, como lâminas decorativas, móveis, construção civil, barcos e outros. Entretanto, é importante considerar que o processamento mecânico de qualquer espécie florestal gera grandes quantidades de resíduos. Assim, os rejeitos resultantes dos processos de laminação e desdobro primário poderiam ser uma fonte de matéria-prima potencial para a produção de vários tipos de produtos reconstituídos de madeira, entre eles os painéis de cimento-madeira.

Este trabalho teve como objetivo avaliar a viabilidade técnica de produção de painéis de cimento-madeira com resíduos da madeira de cedro-australiano (Toona ciliata M. Roem.), bem como a sua mistura com as madeiras de Eucalyptus grandis $x$ Eucalyptus urophylla e Pinus taeda em dois processos distintos de cura.

\section{MATERIAL E MÉTODOS}

\subsection{Matéria-prima}

Para este estudo foram utilizadas madeiras das seguintes espécies: Toona ciliata M. Roem var. australis (18 anos), procedente de um plantio comercial localizado no Município de Marechal Floriano - ES; Pinus taeda (18 anos), procedente de um plantio experimental no Campus da Universidade Federal de Lavras - UFLA, MG; clone do híbrido de Eucalyptus grandis $x$ Eucalyptus urophylla (7 anos), procedente da Companhia Mineira de Metais, localizada no Município de Vazante, MG.

As densidades básicas determinadas para as madeiras de T. ciliata, P. taeda e clone de Eucalyptus foram de 0,$324 ; 0,360 ;$ e $0,560 \mathrm{~g} / \mathrm{cm}^{3}$, respectivamente.
O aglomerante empregado foi o cimento Portland CP V - ARI/Plus, de alta resistência inicial, segundo a Norma Brasileira NBR 5733 (1991). O aditivo químico utilizado foi o cloreto de cálcio $\left(\mathrm{CaCl}_{2} \cdot 2 \mathrm{H}_{2} \mathrm{O}\right)$.

\subsection{Parâmetros do processo}

Para a manufatura dos painéis, procedeu-se, inicialmente, ao cálculo de cada componente (madeira, cimento, água e aditivo), seguindo a metodologia sugerida por Souza (1994). Para a definição da proporção dos componentes na mistura, foram consideradas as seguintes relações: madeira/cimento de 1:2,75; água/ cimento de 1:2,5; taxa de água de hidratação/cimento de $25 \%$ e $4 \%$ de cloreto de cálcio, com base no peso do cimento. Os cálculos foram realizados para uma densidade nominal dos painéis de $1,10 \mathrm{~g} / \mathrm{cm}^{3}$

\subsection{Obtenção e preparação das partículas de madeira}

As partículas de madeira de T. ciliata foram obtidas a partir do aproveitamento dos resíduos da laminação, que foram processados em moinho de martelos. As madeiras de $P$. taeda e de Eucalyptus foram transformadas em pranchões, posteriormente reduzidos em peças com dimensões de $20 \mathrm{~cm}$ de comprimento, $9 \mathrm{~cm}$ de largura e 2,5 cm de espessura. As secções foram reduzidas em partículas de dimensões de 20 x 9,0 x 0,07 cm e, em seguida, processadas em moinho de martelos.

As partículas foram submetidas ao tratamento por imersão em água fria por $24 \mathrm{~h}$, passando, posteriormente, por processos de drenagem e lavagem e, em seguida, secagem ao ar até atingir o teor de umidade de $12 \%$. Posteriormente, realizou-se o peneiramento mecânico para a retirada dos finos.

\subsection{Formação do colchão e prensagem dos painéis}

Os componentes necessários para a formação dos três painéis por tratamento foram pesados e devidamente misturados em betoneira, de eixo inclinado, para a formação da massa homogênea. O colchão foi formado por deposição da massa em caixa formadora com dimensões de 480 × $480 \mathrm{~mm}$, colocada sobre uma chapa de alumínio. Para controle da espessura final dos painéis, foram utilizados espaçadores de ferro de $15 \mathrm{~mm}$ de espessura. Após a distribuição do material, colocou-se outra chapa na face superior do colchão e, em seguida, repetiu-se o procedimento até completar três painéis por tratamento.

Revista Árvore, Viçosa-MG, v.36, n.3, p.549-557, 2012 
Os painéis foram prensados na temperatura ambiente, com pressão específica de $40 \mathrm{kgf} / \mathrm{cm}^{2}$ durante $10 \mathrm{~min}$. A pressão foi mantida até os colchões atingirem a espessura dos separadores, e posteriormente efetivaram-se o grampeamento e liberação da prensa. Os painéis permaneceram grampeados em condições naturais de umidade e temperatura durante $24 \mathrm{~h}$, permitindo que as reações de endurecimento ocorressem para garantir a espessura final desejada. Após esse período, os grampos foram retirados e os painéis, acondicionados de acordo com cada tratamento preestabelecido para maturação.

\subsection{Acondicionamento}

Os painéis cimento-madeira foram submetidos a dois processos distintos de cura. O primeiro consistiu em acondicionar os painéis em câmara climatizada por um período de 28 dias na temperatura de $20 \pm 1^{\circ} \mathrm{C}$ e umidade relativa de $65 \pm 3 \%$. No segundo, optou-se pelo acondicionamento dos painéis em câmara vaporizada na temperatura de $60{ }^{\circ} \mathrm{C}$, durante 10 dias. A escolha do tempo e temperatura foi feita de acordo com os estudos apresentados em Paula et al. (2009). Após os 10 dias, os painéis passaram por uma secagem de $3 \mathrm{~h}$ em estufa com ventilação forçada de ar a uma temperatura de $60{ }^{\circ} \mathrm{C}$ e, em seguida, foram levados para câmara de climatização, completando o período de 28 dias de maturação. Assim, em todos os tratamentos os painéis encerraram seu período de maturação nas mesmas condições.

\subsection{Determinação das propriedades fisicomecânicas}

Após o processo de maturação e climatização dos painéis, foram retirados os corpos-de-prova para ensaios de absorção de água (AA) e inchamento em espessura (IE), depois de 24 h de imersão em água, densidade aparente (DA), módulo de elasticidade (MOE) e módulo de ruptura (MOR) pelo ensaio de flexão estática, compressão paralela (CP) e ligação interna (LI). Para avaliação das propriedades fisicomecânicas dos painéis, adotaram-se os procedimentos recomendados pelas Normas ASTM D-1037 (1999) e DIN 52362 (1982).

\subsection{Delineamento experimental e análise estatística}

O delineamento experimental foi inteiramente casualizado e disposto em esquema fatorial com dois fatores $(3 \times 2)$, referentes a três composições e dois processos de maturação, com três repetições de cada tratamento, totalizando 18 painéis (Tabela 1 ).

Os resultados dos ensaios físicos e mecânicos foram submetidos à análise de variância a $5 \%$ de significância, para avaliar o efeito dos tratamentos (composição e maturação) e suas interações. As médias foram comparadas pelo teste de Tukey a 5\% de significância.

\section{RESULTADOS}

\subsection{Propriedades físicas}

Os valores médios das propriedades físicas, DA e AA após 24 h de imersão não apresentaram interação significativa entre os fatores cura e composição e estão apresentados na Tabela 2 .

A densidade aparente dos painéis sofreu influência somente do fator composição, e os painéis compostos pela mistura de T. ciliata e Eucalyptus apresentaram média estatisticamente superior aos demais. A absorção de água foi influenciada pelo tipo de cura, sendo a média dos painéis submetidos à cura a vapor inferior à dos painéis acondicionados em câmara climática.

Tabela 1 - Delineamento experimental.

Table 1 - Experimental design.

\begin{tabular}{|c|c|c|c|c|}
\hline \multirow{2}{*}{ Tratamento } & \multicolumn{3}{|c|}{ Proporção das espécies, \% } & \multirow{2}{*}{ Maturação } \\
\hline & T. ciliata & Pinus taeda & E. grandis $x$ E. urophylla & \\
\hline $\mathrm{T} 1-\mathrm{T} / \mathrm{Cc}$ & 100 & - & - & Climatização \\
\hline $\mathrm{T} 2-\mathrm{TP} / \mathrm{Cc}$ & 50 & 50 & - & Climatização \\
\hline $\mathrm{T} 3-\mathrm{TE} / \mathrm{Cc}$ & 50 & - & 50 & Climatização \\
\hline $\mathrm{T} 4-\mathrm{T} / \mathrm{Cv}$ & 100 & - & - & Vaporização \\
\hline $\mathrm{T} 5-\mathrm{TP} / \mathrm{CV}$ & 50 & 50 & - & Vaporização \\
\hline $\mathrm{T} 6-\mathrm{TE} / \mathrm{CV}$ & 50 & - & 50 & Vaporização \\
\hline
\end{tabular}

T: Toona ciliata M. Roem.; P: Pinus taeda; E: E. grandis x E. urophylla; Cc: Câmara climática; e Cv: Câmara de vaporização.

Revista Árvore, Viçosa-MG, v.36, n.3, p.549-557, 2012 
Para inchamento em espessura, após 24 h de imersão em água a interação entre os fatores cura e composição foi significativa, e a análise do desdobramento dessa propriedade está apresentada na Tabela 3. Analisando as diferentes composições dos painéis curados em câmara climática, verificou-se que o tratamento composto pela mistura de T. ciliata e Eucalyptus apresentou maior inchamento em espessura. Para a cura a vapor não houve diferença significativa entre as composições. Para o fator cura, somente o tratamento composto por T. ciliata e Eucalyptus apresentou diferença significativa entre os dois processos de cura, tendo os painéis curados em câmara climática a maior média.

\subsection{Propriedades mecânicas}

MOE, MOR e CP não apresentaram interações significativas entre os fatores cura e composição e estão apresentados na Tabela 4. Verifica-se, nessa Tabela, que ambos os fatores, isoladamente, influenciaram significativamente o MOE e o MOR, entretanto a CP foi influenciada significativamente somente pelo fator cura.

Tabela 2 - Valores médios das propriedades físicas dos painéis cimento-madeira de acordo com sua composição e processo de cura.

Table 2 - Physical properties average values of the cement-wood panels according to the their composition and cure procedure.

\begin{tabular}{|c|c|c|}
\hline Fatores & Densidade aparente & AA_24h \\
\hline Composição & ---- g/cm³ --- & -------- \% --------- \\
\hline $\mathrm{T}$ & $1,13 \mathrm{~B}$ & $13,0 \mathrm{~A}$ \\
\hline TP & $1,15 \mathrm{~B}$ & $12,4 \mathrm{~A}$ \\
\hline TE & $1,21 \mathrm{~A}$ & $12,4 \mathrm{~A}$ \\
\hline F calculado & $14,425 *$ & $0,509^{\mathrm{ns}}$ \\
\hline \multicolumn{3}{|l|}{ Cura } \\
\hline $\mathrm{Cc}$ & $1,17 \mathrm{~A}$ & $13,5 \mathrm{~A}$ \\
\hline $\mathrm{Cv}$ & $1,16 \mathrm{~A}$ & $11,6 \mathrm{~B}$ \\
\hline F calculado & $0,478^{\mathrm{ns}}$ & $12,869^{*}$ \\
\hline CV (\%) & 2,34 & 9,21 \\
\hline
\end{tabular}

Médias seguidas de mesma letra não diferem estatisticamente entre si, pelo teste de Tukey a 5\% de significância. T: Toona ciliata M. Roem.; P: Pinus taeda; E: E. grandis x E. urophylla; Cc: Câmara climática; Cv: Câmara de vaporização; e AA_24h: Absorção de água após 24 h de imersão.

e": Valor de F significativo a 5\% de significância; ens: valor de F não significativo a 5\% de significância; e C.V.: Coeficiente de variação.

Tabela 3 - Análise do desdobramento da composição dentro de cura para inchamento em espessura após 24 h imersas em água.

Table 3 - Interaction between the panels composition and cure for thickness swelling after 24 hours of immersion.

\begin{tabular}{|c|c|c|c|}
\hline \multirow{2}{*}{ Composição/cura } & \multicolumn{2}{|c|}{ IE_24h } & \multirow{2}{*}{ F calculado } \\
\hline & $\mathrm{Cc}$ & $\mathrm{Cv}$ & \\
\hline \multicolumn{4}{|c|}{ 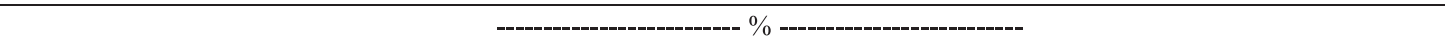 } \\
\hline $\mathrm{T}$ & $0,73 \mathrm{Ba}$ & $0,52 \mathrm{Aa}$ & $4,546^{\mathrm{ns}}$ \\
\hline $\mathrm{TP}$ & $0,74 \mathrm{Ba}$ & $0,57 \mathrm{Aa}$ & $0,211^{\mathrm{ns}}$ \\
\hline TE & $1,57 \mathrm{Aa}$ & $0,69 \mathrm{Ab}$ & $96,199 *$ \\
\hline F calculado & $45,015^{*}$ & $1,513^{\text {ns }}$ & $\begin{array}{ll}------- \\
\end{array}$ \\
\hline $\mathrm{CV}(\%)=15,49$ & & & \\
\hline
\end{tabular}

Médias seguidas de mesma letra maiúscula na coluna não diferem, estatisticamente, pelo teste de Tukey a 5\% de significância. Médias seguidas de mesma letra minúscula na linha não diferem entre si, pelo teste de Tukey a 5\% de significância. T: Toona ciliata M. Roem; P: Pinus taeda; E: E. grandis $x$ E. urophylla; Cc: Câmara climática; Cv: Câmara de vaporização; e IE_24h: Inchamento em espessura após $24 \mathrm{~h}$ de imersão em água.

e": Valor de F significativo a 5\% de significância; e ${ }^{\text {ns: }}$ valor de F não significativo a 5\% de significância; e C.V.: Coeficiente de variação. 
Analisando o fator composição, os painéis de $T$. ciliata foram estatisticamente superiores aos painéis confeccionados pela mistura de $T$. ciliata com Eucalyptus para as propriedades MOE e MOR.

Ainda na Tabela 4, observa-se que o MOE, o MOR e a compressão paralela sofreram influências do fator cura, portanto os painéis acondicionados em câmara de vaporização durante 10 dias apresentaram propriedades inferiores aos painéis curados em câmara climática por 28 dias.

Para ligação interna, a interação entre os fatores cura e composição foi significativa, e a análise do desdobramento dessa propriedade está apresentada na Tabela 5 .

Os valores médios obtidos a partir dos painéis de diferentes composições e curados em câmara climática não apresentaram diferenças significativas entre si, pelo teste de Tukey $(\mathrm{P} \leq 0,05)$. Para a cura a vapor, somente os painéis produzidos com $T$. ciliata exibiram valor estatisticamente superior ao das demais composições. Para o fator cura, somente o tratamento composto pela mistura de T. ciliata e Eucalyptus mostrou diferença significativa entre os dois processos de cura, sendo o valor obtido pela cura em câmara climática estatisticamente superior à média obtida pela cura a vapor.

\section{DISCUSSÃO}

\subsection{Propriedades físicas}

Os resultados de DA não deveriam ter sofrido influência do fator composição, visto que a densidade aparente nominal estabelecida no plano experimental foi de $1,10 \mathrm{~g} / \mathrm{cm}^{3}$. Possivelmente deve ter ocorrido subestimação da umidade das partículas de Eucalyptus, provocando erro nos cálculos de cada componente do colchão dos Tratamentos 3 e 6. Resultados diferentes dos valores de densidade em relação ao predeterminado também foram encontrados por outros autores como Lopes et al. (2005) e Okino et al. (2004).

Os resultados de AA obtidos neste estudo são inferiores a alguns encontrados na literatura para painéis de cimento-madeira, produzidos com as espécies de reflorestamento mais utilizadas, como pinus e eucalipto. Iwakiri e Prata (2008), utilizando as espécies Eucalyptus grandis, Eucalyptus dunnii e Pinus taeda na manufatura de painéis cimento-madeira, encontraram valores de absorção de água, após $24 \mathrm{~h}$ de imersão, de 15,2; 16,7; e 14,0\%, respectivamente. Latorraca (2000), estudando a influência de quatro espécies de eucalipto, encontrou, na mesma propriedade, valores médios entre 15,7 e $22,2 \%$.

Tabela 4 - Valores médios das propriedades mecânicas dos painéis cimento-madeira de acordo com sua composição e processo de cura.

Table 4 -Mechanical properties average values of the cement-wood panels according to the their composition and cure procedure.

\begin{tabular}{|c|c|c|c|}
\hline \multirow{2}{*}{ Fatores } & \multicolumn{2}{|c|}{ Flexão estática } & \multirow{2}{*}{ Compressão paralela } \\
\hline & MOE & MOR & \\
\hline Composição & \multicolumn{3}{|c|}{ 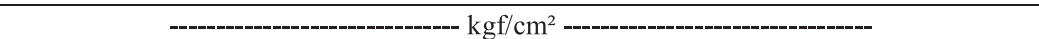 } \\
\hline $\mathrm{T}$ & $46.215 \mathrm{~A}$ & $84,50 \mathrm{~A}$ & $106,88 \mathrm{~A}$ \\
\hline $\mathrm{TP}$ & $44.086 \mathrm{AB}$ & $79,29 \mathrm{~A}$ & $106,12 \mathrm{~A}$ \\
\hline $\mathrm{TE}$ & $39.113 \mathrm{~B}$ & $56,92 \mathrm{~B}$ & $95,10 \mathrm{~A}$ \\
\hline F calculado & $4,432 *$ & $55,557^{*}$ & $1,698^{\mathrm{ns}}$ \\
\hline \multicolumn{4}{|l|}{ Cura } \\
\hline $\mathrm{Cc}$ & $45.391 \mathrm{~A}$ & $80,03 \mathrm{~A}$ & $114,92 \mathrm{~A}$ \\
\hline $\mathrm{Cv}$ & $40.885 \mathrm{~B}$ & $67,11 \mathrm{~B}$ & $90,48 \mathrm{~B}$ \\
\hline F calculado & $5,080^{*}$ & $32,369^{*}$ & $17,510^{*}$ \\
\hline CV (\%) & 9,83 & 6,55 & 12,07 \\
\hline
\end{tabular}

Médias seguidas de mesma letra não diferem estatisticamente entre si, pelo teste de Tukey a 5\% de significância; T: Toona ciliata M. Roem.; P: Pinus taeda; E: E. grandis x E. urophylla; Cc: Câmara climática; Cv: Câmara de vaporização; MOE: módulo de elasticidade; e MOR: módulo de ruptura

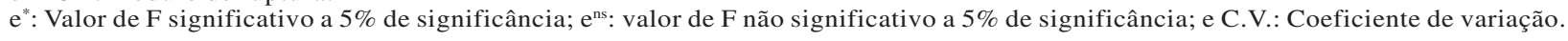

Revista Árvore, Viçosa-MG, v.36, n.3, p.549-557, 2012 
Tabela 5 - Interação entre composição e cura dos painéis para ligação interna.

Table 5 - Interaction between the panels composition and cure for internal bonding.

\begin{tabular}{|c|c|c|c|}
\hline & \multicolumn{2}{|c|}{ Ligação Interna } & \multirow{2}{*}{ F calculado } \\
\hline & $\mathrm{Cc}$ & $\mathrm{Cv}$ & \\
\hline & \multicolumn{2}{|c|}{---------------------- kgf/cm²--------------------- } & \\
\hline $\mathrm{T}$ & $10,08 \mathrm{~A} \mathrm{a}$ & $11,49 \mathrm{~A} \mathrm{a}$ & $2,249^{\text {ns }}$ \\
\hline TP & $10,32 \mathrm{~A} \mathrm{a}$ & $8,54 \mathrm{~B} \mathrm{a}$ & $3,601^{\mathrm{ns}}$ \\
\hline TE & $10,40 \mathrm{~A} \mathrm{a}$ & $8,30 \mathrm{~B} \mathrm{~b}$ & $5,028^{*}$ \\
\hline F calculado & $0,063^{\text {ns }}$ & $7,167^{*}$ & $\begin{array}{l}------ \\
\end{array}$ \\
\hline $\mathrm{CV}(\%)=8,76$ & & & \\
\hline
\end{tabular}

Médias seguidas de mesma letra maiúscula na coluna não diferem, estatisticamente, pelo teste de Tukey a 5\% de significância. Médias seguidas de mesma letra minúscula na linha não diferem entre si, pelo teste de Tukey a 5\% de significância. T: Toona ciliata M. Roem; P: Pinus taeda; E: E. grandis x E. urophylla; Cc: Câmara climática; e Cv: Câmara de vaporização.

$\mathrm{e}^{*}$ : Valor de F significativo a 5\% de significância; $\mathrm{e}^{\text {ns: }}$ : valor de F não significativo a 5\% de significância; e C.V.: Coeficiente de variação.

Os valores obtidos neste estudo para IE após 24 h de imersão em água são compatíveis na faixa de 1,50 a $1,80 \%$, estabelecida por Viroc (2004) para chapas comerciais e na faixa mínima de 1,2 a $1,8 \%$, admitida pelo processo Bison Wood-Cement Board (1978). Sobre valores referenciados na literatura, Semple et al. (2002) encontraram um valor médio de $4,4 \%$ para inchamento em espessura após $24 \mathrm{~h}$ em painéis confeccionados com resíduos de eucalipto, sem a inclusão de casca.

O inchamento em espessura é uma das propriedades mais importantes dos painéis cimento-madeira e os coloca em vantagem em relação a outros tipos de painéis reconstituídos de madeira quando empregados em ambientes com variações de umidade. Segundo Lee (1984), essa alta estabilidade dimensional ocorre em razão de fatores como diversos espaços que permitem o inchamento interno e o revestimento do cimento ao redor da partícula, restringindo o seu inchamento.

\subsection{Propriedades mecânicas}

Os valores de MOE obtidos neste estudo estão acima de alguns encontrados na literatura e do valor mínimo de $30.000 \mathrm{kgf} / \mathrm{cm}^{2}$, exigido pelo processo Bison WoodCement Board (1978). Santos et al. (2008) obtiveram valores médios de 38.503 e $35.953 \mathrm{kgf} / \mathrm{cm}^{2}$, respectivamente, em painéis de candeia associados ao eucalipto e ao pinus, com proporção de mistura de 50\%. Latorraca e Iwakiri (2000) conseguiram valores para MOE variando de 15.176 a $44.005 \mathrm{kgf} / \mathrm{cm}^{2}$ em painéis de Eucalyptus dunnii com densidade nominal de $1,2 \mathrm{~g} / \mathrm{cm}^{3}$.
Embora os valores de MOR encontrados neste estudo estejam abaixo do exigido pelo processo Bison Wood-Cement Board (1978), que está em torno de $90 \mathrm{kgf} / \mathrm{cm}^{2}$, eles são compatíveis com aqueles encontrados na literatura. Latorraca (2000), estudando as propriedades de painéis confeccionados com diferentes espécies de eucalipto, obteve valores variando de 36,0 a $69,6 \mathrm{kgf} / \mathrm{cm}^{2}$. Okino et al. (2004) encontraram valores entre 43,67 e 79,15 kgf/cm² em painéis confeccionados com quatro clones de seringueira, com densidade nominal de $1,4 \mathrm{~g} / \mathrm{cm}^{3}$. Matosk (2005) conseguiu valores de 51 a $60,35 \mathrm{kgf} / \mathrm{cm}^{2}$ para painéis confeccionados a partir de pó de pinus e $\mathrm{CaCl}_{2}$ como aditivo químico.

Os valores de compressão paralela obtidos neste estudo foram compatíveis com a literatura. Latorraca (2000), avaliando as propriedades de painéis cimentomadeira confeccionados com quatro espécies de eucalipto, encontrou valor médio de $66,86 \mathrm{kgf} / \mathrm{cm}^{2}$ para resistência à compressão paralela. Santos et al. (2008) obtiveram, para a mesma propriedade, valores médios de 62 e $43 \mathrm{kgf} / \mathrm{cm}^{2}$, em painéis confeccionados com Eremanthus erytropappus associada ao eucalipto e ao pinus, respectivamente, na proporção de $50 \%$.

Os valores médios obtidos para ligação interna foram satisfatoriamente superiores aos encontrados na literatura e ao valor mínimo requerido pelo processo Bison Wood-cement Board (1978), que é de $4,0 \mathrm{kgf} / \mathrm{cm}^{2}$. Silva et al. (2005), estudando compósitos de madeira e casca de Eucalyptus urophylla, encontraram valores entre 3,34 e $6,98 \mathrm{kgf} / \mathrm{cm}^{2}$. Santos et al. (2008) 
obtiveram para resistência à ligação interna valores de 6,13 e $6,11 \mathrm{kgf} / \mathrm{cm}^{2}$, em painéis confeccionados com resíduos de Eremanthus erytropappus associada ao pinus e ao eucalipto, respectivamente.

A influência do processo de cura a vapor sobre as propriedades mecânicas de painéis cimento-madeira já foi estudada por Latorraca e Iwakiri (2001). Esses autores concluíram que em algumas propriedades mecânicas os painéis submetidos a esse processo, durante 3 e 10 dias, não diferiram estatisticamente dos painéis curados em câmara climática durante 28 dias. Para painéis curados a vapor durante 10 dias, encontraram-se valores de $28.652,58$ e $51,35 \mathrm{kgf} / \mathrm{cm}^{2}$ para MOE e compressão paralela, respectivamente, ou seja, inferiores aos deste estudo. Para as propriedades MOR e ligação interna, esse processo não proporcionou aumento da resistência dos painéis. Esses autores relataram que a prolongada exposição dos painéis ao calor pode ter provocado transformações na composição química da madeira de modo a afetar negativamente a interação cimento-madeira.

\section{CONCLUSÕES}

Os resultados obtidos neste estudo indicaram a viabilidade técnica da madeira de $T$. ciliata como fonte de matéria-prima alternativa na confecção de painéis de madeira-cimento, seja como espécie principal, seja associada às madeiras de $P$. taeda e Eucalyptus.

De modo geral, as propriedades fisicomecânicas dos painéis de cimento-madeira apresentaram valores expressivos e compatíveis em relação aos referenciados na literatura e superiores em comparação com as normas correspondentes.

Com relação os processos de maturação adotados, a cura a vapor promoveu melhores resultados às propriedades físicas, enquanto na maioria das propriedades mecânicas ela afetou negativamente a resistência dos painéis.

Quanto à composição, os painéis compostos pela mistura de T. ciliata com Eucalyptus apresentaram valores médios superiores para inchamento em espessura e resultados inferiores para a maioria das propriedades mecânicas, indicando uma possível influência negativa do Eucalyptus sobre a resistência das chapas.

\section{REFERÊNCIAS}

AMERICAN SOCIETY FOR TESTING AND

MATERIAL - ASTM. ASTM D 1037 -

Standard methods of evaluating the properties of wood-base fiber and particle panel materials. Philadelphia: 1999.

ASSOCIAÇÃOBRASILEIRADENORMAS TÉCNICAS - ABNT. NBR 5733 Cimento Portland de alta resistência inicial. Rio de Janeiro: 1991. 5p.

BERALDO, A. L.; SAMPAIO, C. A. P. Compósitos à base de resíduos vegetais e cimento Portland. In: ENCONTRO BRASILEIRO EM MADEIRA E ESTRUTURAS DE MADEIRA, 8., 2002,

Uberlândia-MG. Anais... Uberlândia, MG: FECIV/ UFU, 2002. p.120-126.

\section{BISON WOOD-CEMENT BOARD. Bison -} Report 1978.p.10.

BYGRAVE, F. L.; BYGRAVE, P. L. Growing Australian Red Cedar. Canberra: RIRDC/ Land \& Water Australia/FWPRDC/MDBC Joint Venture Agroforestry Program, 2005. 84p.

HERRERA, R. E.; CLOUTIER, A. Compatibility of four eastern Canadian woods with gypsum and gypsum-cement binders by isothermal calorimetry. Maderas. Ciencia y Tecnología, v.10, n.3, p.275-288, 2008.

IWAKIRI, S.; PRATA, J. G. Utilização da madeira de Eucalyptus grandis e Eucalyptus dunnii na produção de painéis cimento-madeira. Cerne, v.14, n.1, p.68-74, 2008.

\section{LATORRACA, J. V. F. Eucalyptus spp. na produção de painéis de cimento madeira. 2000. 191f. Tese (Doutorado em Ciências Florestais) - Universidade Federal do Paraná, Curitiba, 2000.}

LATORRACA, J. V. F.; IWAKIRI, S. Efeito da cura a vapor sobre as propriedades mecânicas de painéis de cimento-madeira. Floresta e Ambiente, v.8, n.1, p.84-93, 2001.

LATORRACA, J. V. F.; IWAKIRI, S. Efeitos do tratamento das partículas de Eucalyptus dunnii (maid), da variação da relação madeira-cimento e do uso de aditivos sobre as propriedades físicas e mecânicas de painéis de madeira-cimento. Cerne, v.6, n.1, p.68-76, 2000. 
LEE, A. W. C. Physical and mechanical properties of cement bonded southern pine excelsior board. Forest Products Journal, v.34, n.4, p.30-34, 1984.

LIMA, N. N. et al. Avaliação do tempo de cura sobre as propriedades dos painéis cimento-madeira de faveira (Parkia gigantocarpa Ducke). In: SIMPÓSIO DE PÓS-GRADUAÇÃO EM CIÊNCIAS FLORESTAIS, 6.; SIMPÓSIO DE CIÊNCIAE TECNOLOGIA DA MADEIRA DO RJ, 2., 2010, Rio de Janeiro. Anais... Rio de Janeiro: 2010.

LOPES, Y. L. V. et al. Avaliação do potencial técnico da madeira e cascas de Eucalyptus grandis Hill ex Maiden na produção de painéis cimento-madeira. Scientia Forestalis. n.67, p.111-122, 2005.

MATOSK, A. Utilização de pó de madeira com granulometria controlada na produção de painéis de cimentomadeira. 2005. 202f. Tese (Doutorado em Ciências Florestais) - Universidade Federal do Paraná, Curitiba, 2005.

MOSLEMI, A. A.; GARCIA, J. F.; HOFSTRAND, A. D. Effect of various treatments and additives on wood-portland cementwater systems. Wood and Fiber Science, v.15, n.2, p.164 -176, 1983.

\section{NORMEN FÜR HOLZFASERPLATEN}

SPANPLATTEN SPERRHOLZ. - DIN 52362 - Testing of wood chipboards, bending test, determination of bending strength. Germany: 1982. p.39-40.

OKINO, E. Y. A. et al. Chapa aglomerada de cimento-madeira de Hevea brasiliensis müll. Revista Árvore, v.28, n.3, p.451-457, 2004.

PAPADOULOS, A. N. Decay resistance of cementbonded oriented strand board. Bioresources, v.1, n.1, p.62-66, 2006.
PAULA, L. E. R. et al. Efeito, p. da temperatura e tempo de vaporização na cura de painéis cimento madeira. Cerne. v.15, n.4, p.439-445, 2009.

PINHEIRO, A. L.; LANI, J. L.; COUTO, L. CedroAustraliano: cultivo e utilização (Toona ciliata M. Roem. var. australis (F. Muell) Bahadur. Viçosa, MG: Universidade Federal de Viçosa, 2006. 42p.

SANTOS, R. C. et al. Aproveitamento de resíduos da madeira de candeia (Eremanthus erythropappus) para produção de painéis cimentomadeira. Cerne, v.14, n.3, p.241-250, 2008.

SAVASTANO JÚNIOR, H.; WARDEN, P. G.; COUTTS, R. S. P. Brazilian waste fibers as reinforcement of cement-based composites. Cement and Concrete Composites, v.22, n.25, p.379-384, 2000.

SEMPLE, K. E.; CUNNINGHAM, R. B.; EVANS, M. P. D. The suitability of Five Western Australian mallee eucalypt species for wood-cement composites. Industrial Crops and Products, v. 16, n.2, p.89-100, 2002 .

SILVA, G. C. et al. Produção do compósito madeira e casca de Eucalyptus urophylla S.T. Blake e cimento Portland. Scientia Forestalis, n.68, p.59-67, 2005.

SOUZA, M. R. Durability of cementbonded particleboard made conventionally and carbon dioxide injection. 1994. 123f. Tese (Doctor of Philosophy) - University of Idaho, Idaho, 1994.

VIROC. Características Viroc. 2004. Disponível em: <http://www.viroc.pt> Acesso em: 23 jul. 2009. 